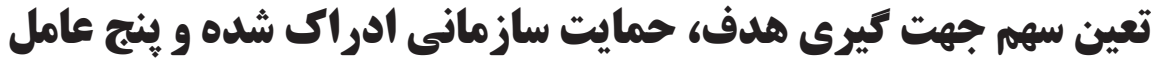

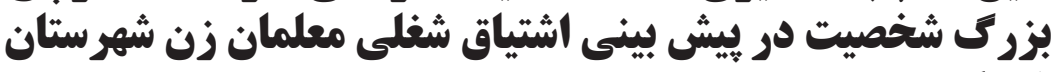

\author{
مهرداد حاجى حسنى '“":، منيزه كاوه'
}

' استاديار، كروه مشاوره، دانشخاه شهركرد، شهركرد، ايران

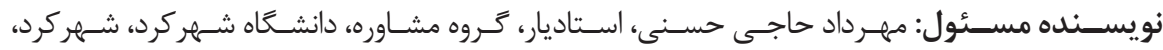
ايـران. ايميـل: mehrdadhajihasani@gmail.com

DOI: $10.21859 /$ joe- 04031

קقكيده

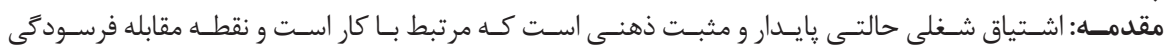

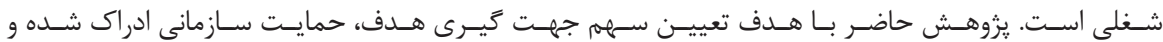

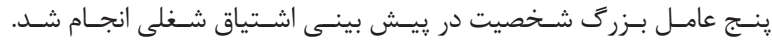

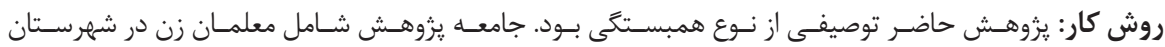

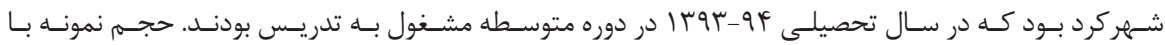

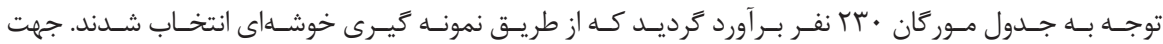

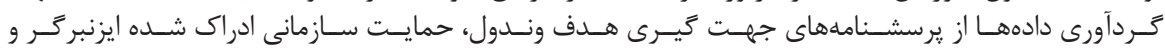

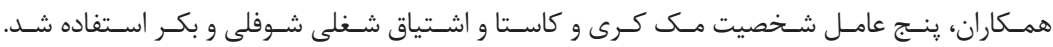

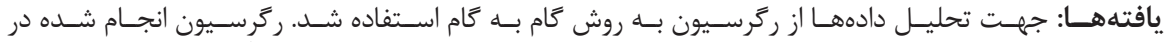

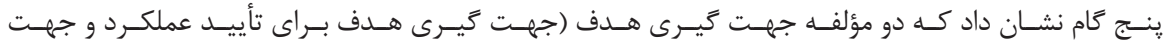

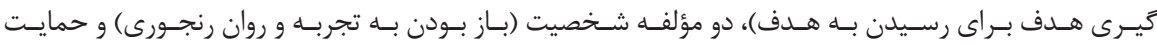

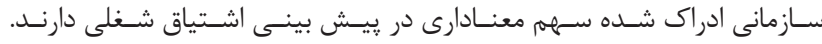

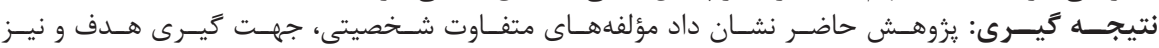

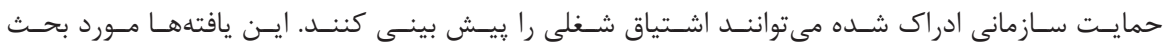

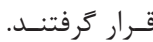

تاريخ دريافت مقاله: | |r/T/

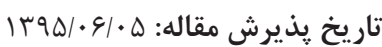

وازَّان كليدى:

جهت كيرى هدف حمايت سازمانى ادرى شده هدف ينج عامل شخصيت

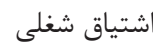

تمامى حقوق نشر براى دانشعاه علوم يزشكى همدان محفوظ است.

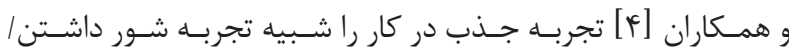

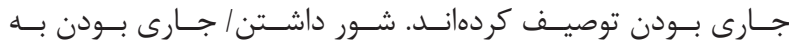

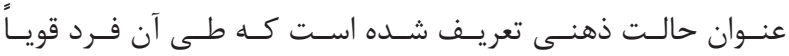

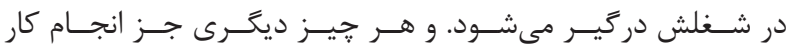

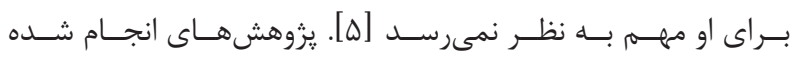

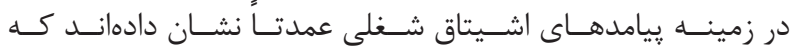

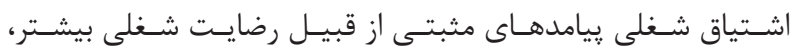

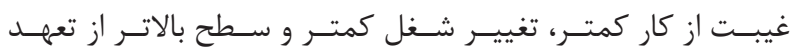

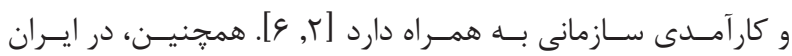

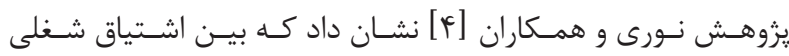

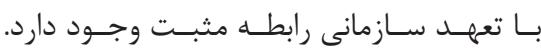

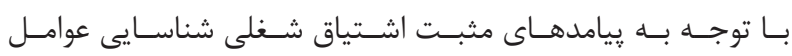

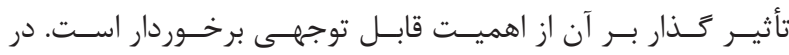

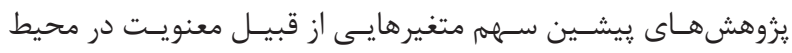

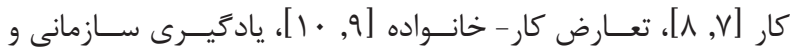

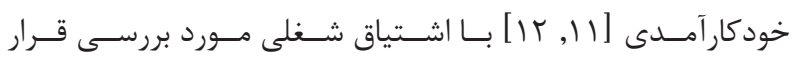

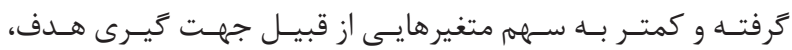

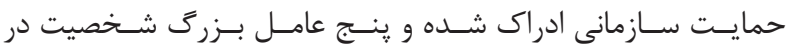

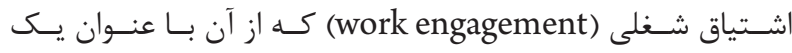

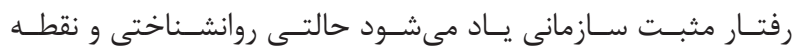

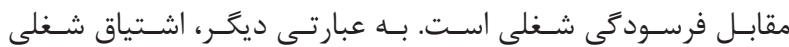

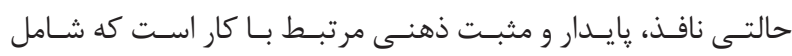

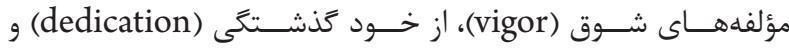

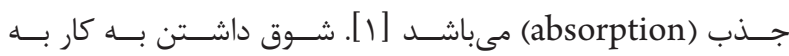
ســح بـالاى انــرزى و تساب آورى ذهنـى در حيـن كار اشـاره دارد

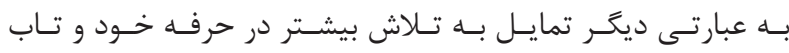

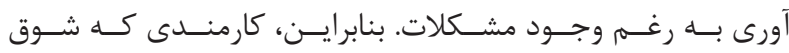

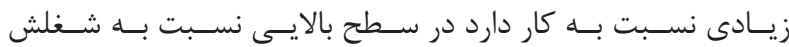

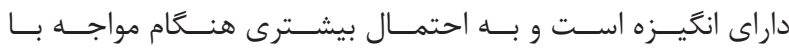

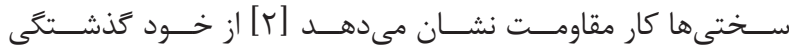

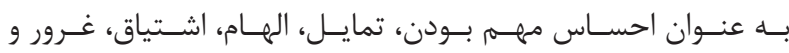

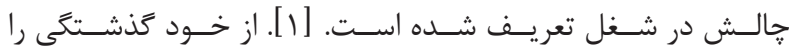

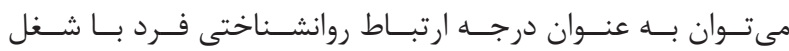

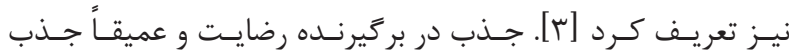

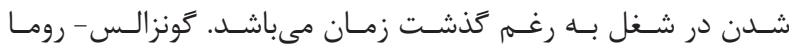




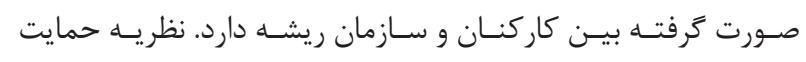

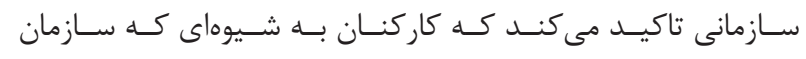

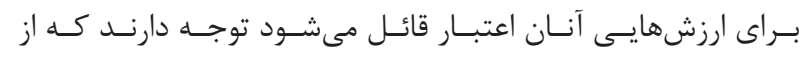

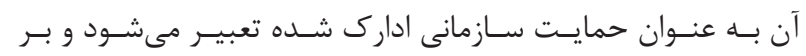

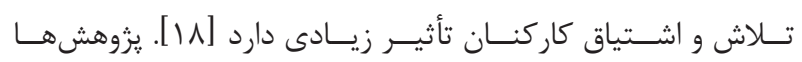

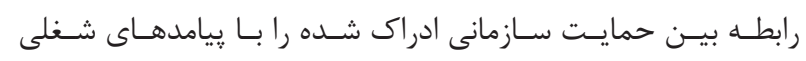

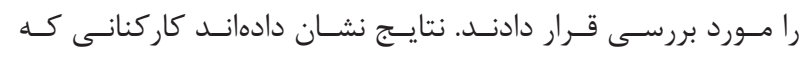

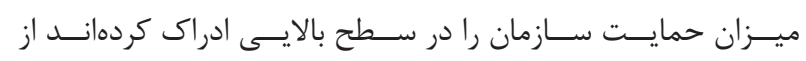

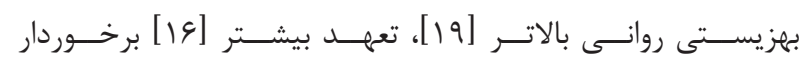

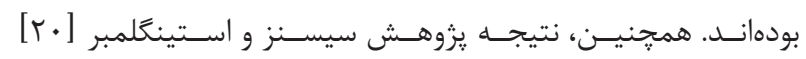

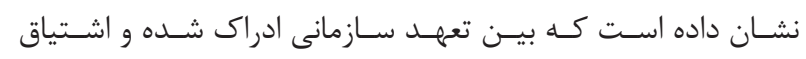

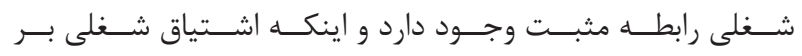

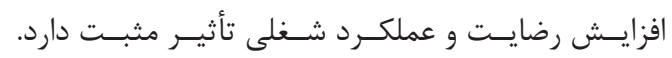

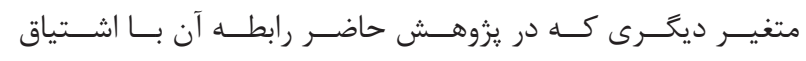

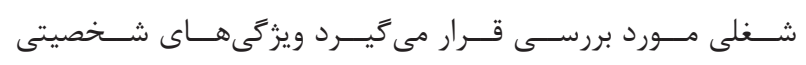

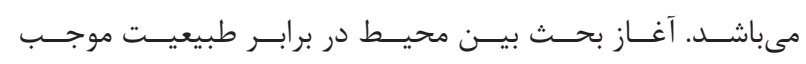

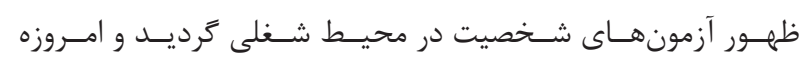

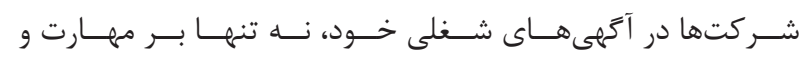

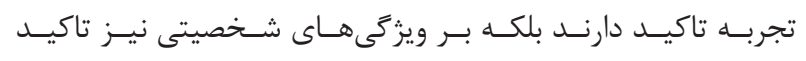

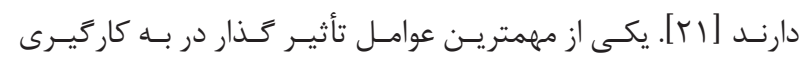

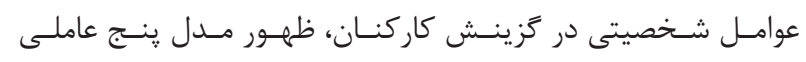

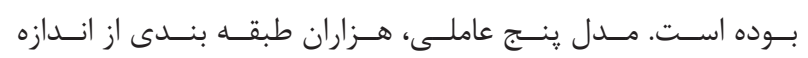

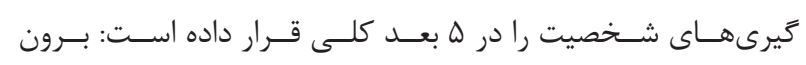

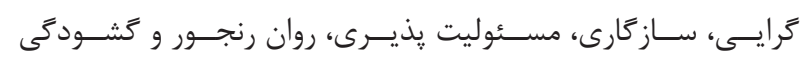

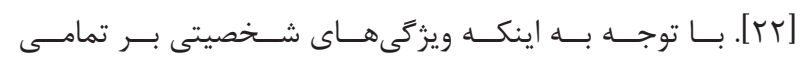

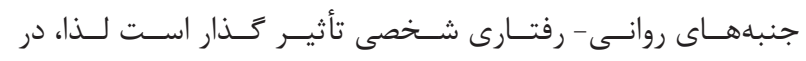

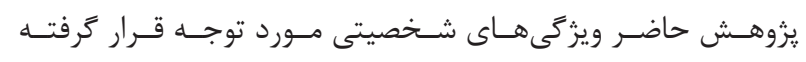

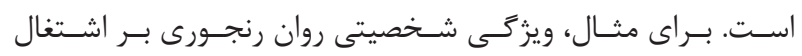

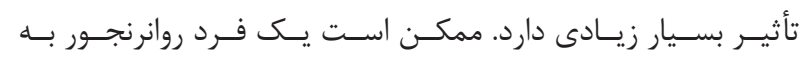

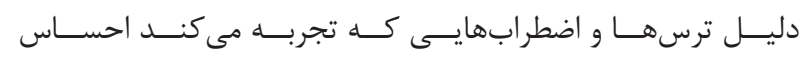

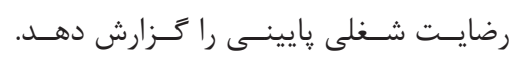

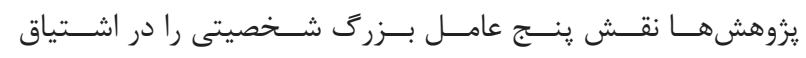

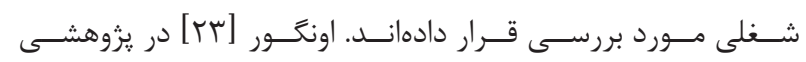

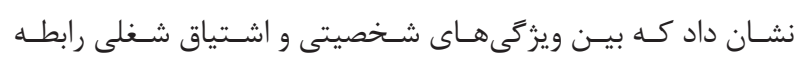

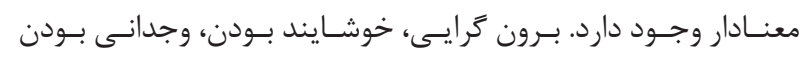

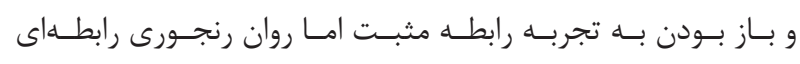

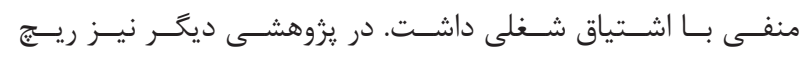

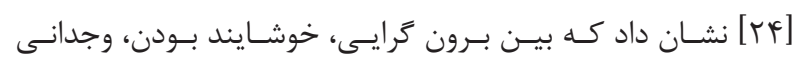

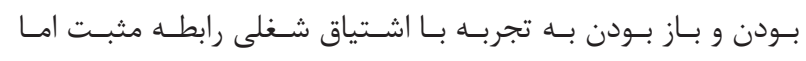

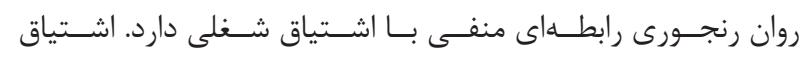

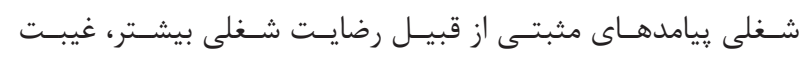

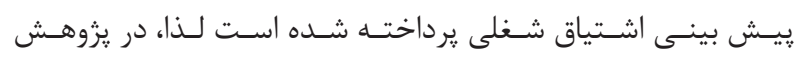

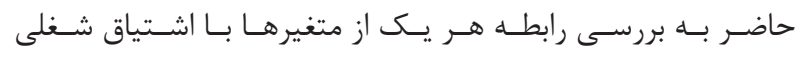

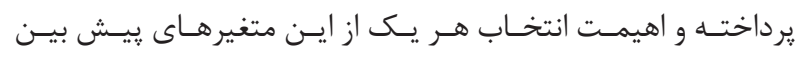

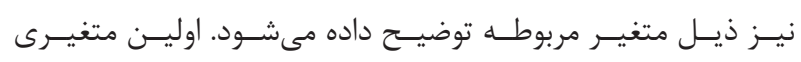

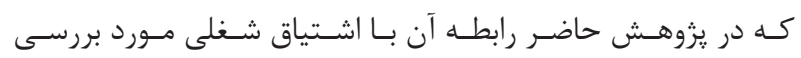

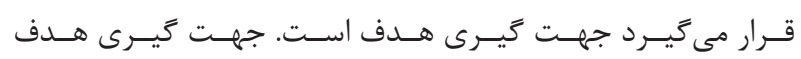

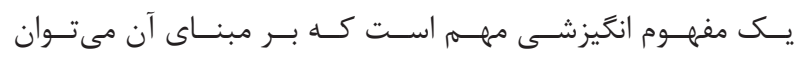

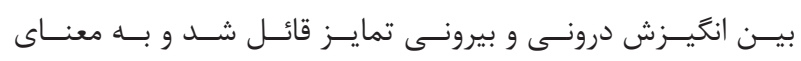

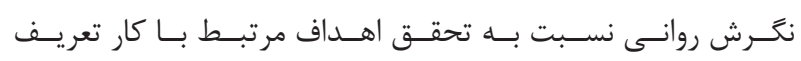

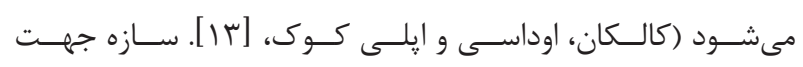

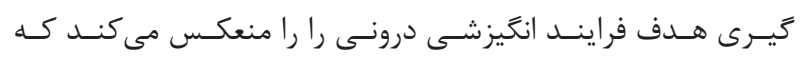

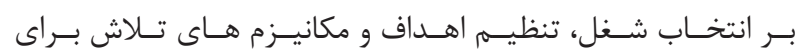

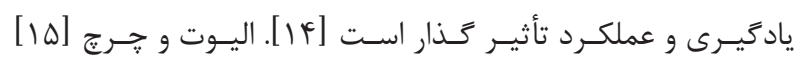

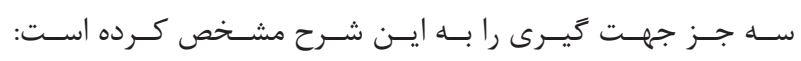

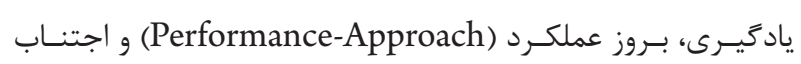

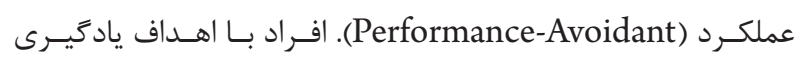

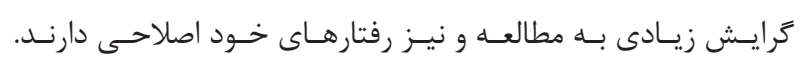

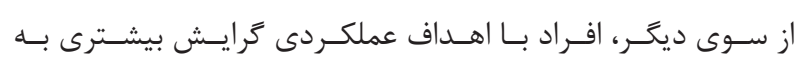

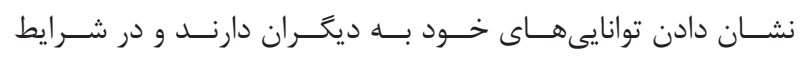

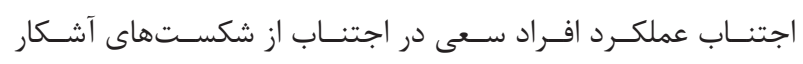

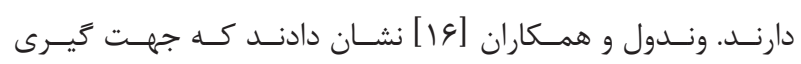

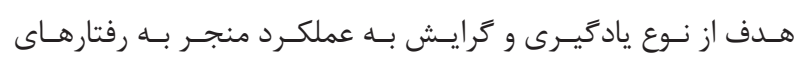

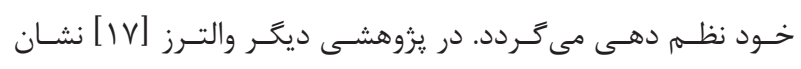

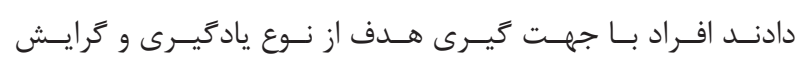

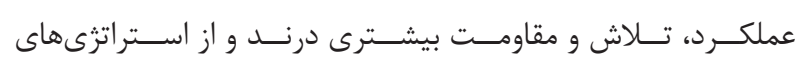

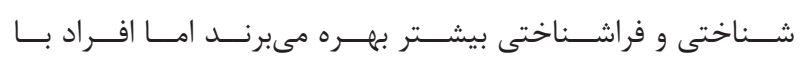

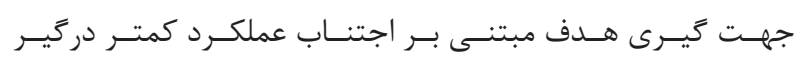

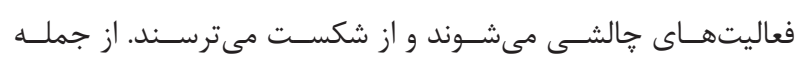

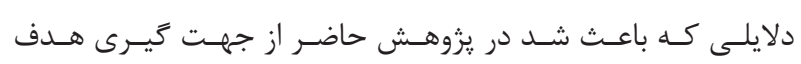

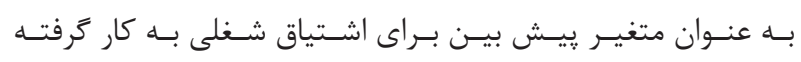

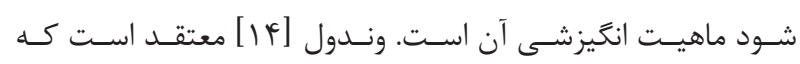

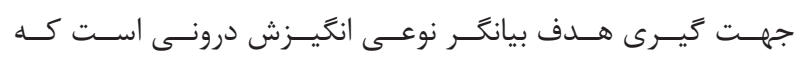

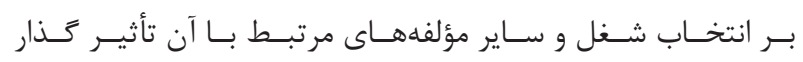

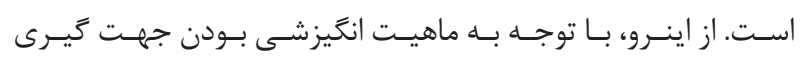

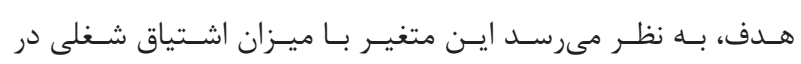
ارتبــاط باشـد.

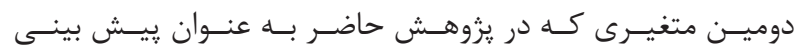

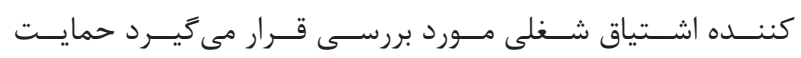
سـازمانى ادراى شــــ (perceived organizational support)

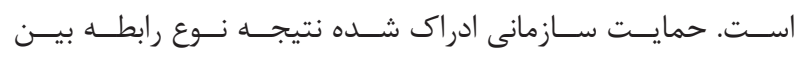

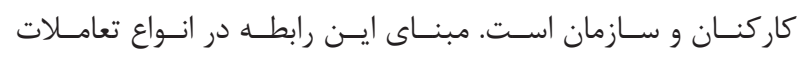


ץ (كمـى مخالفــم)، \& (كمــى موافقــم)، ه (موافقــم) و 9 (كامــلاً

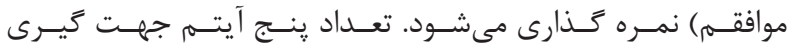

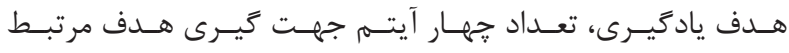

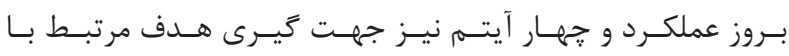

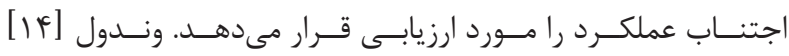

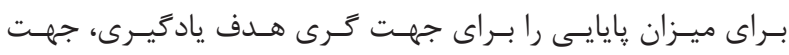

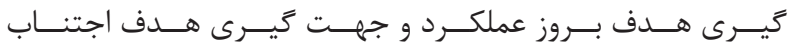

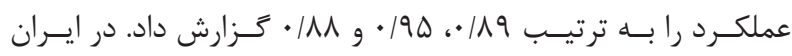

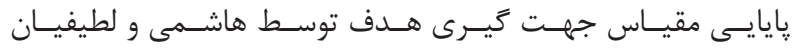

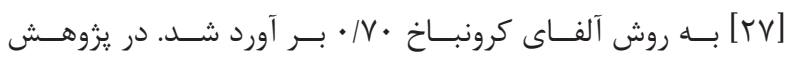

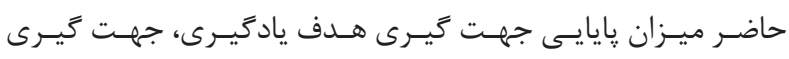

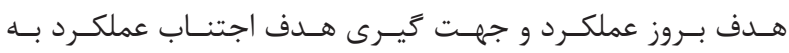

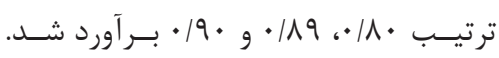

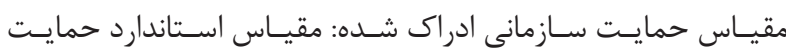

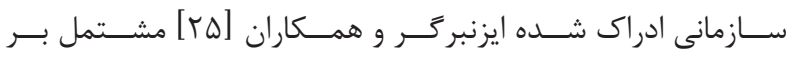

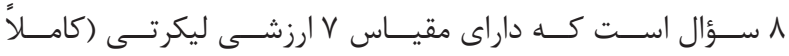

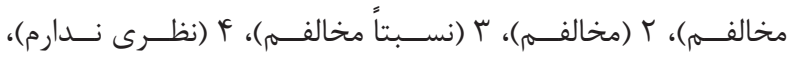

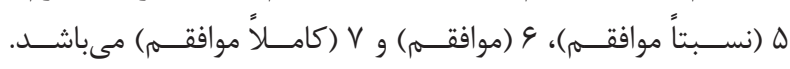

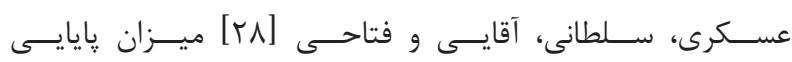

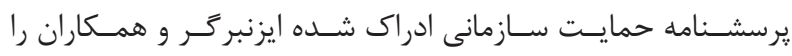

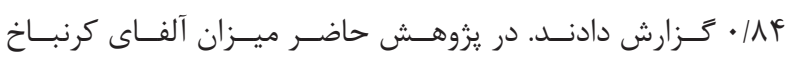

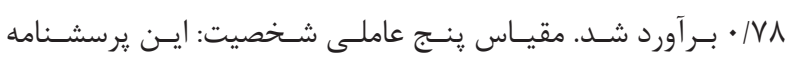

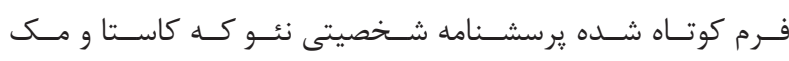

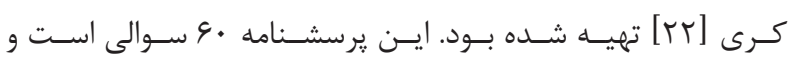

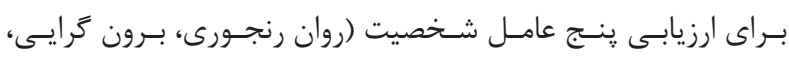

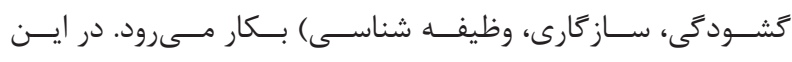

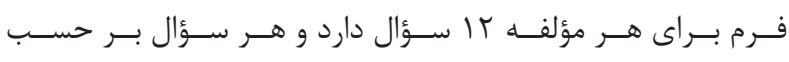

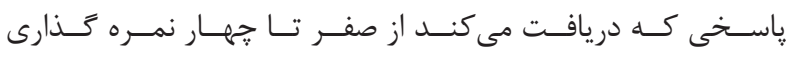

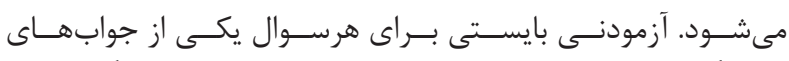

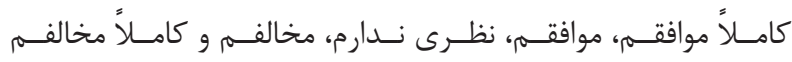

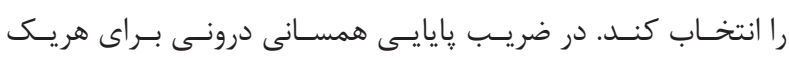

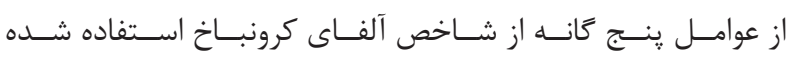

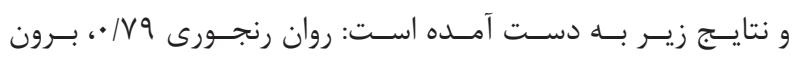

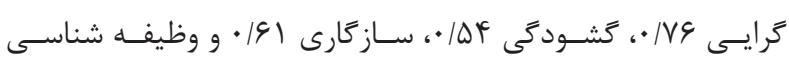

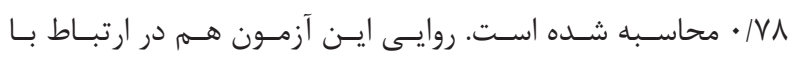

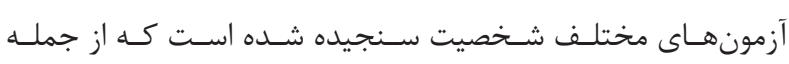

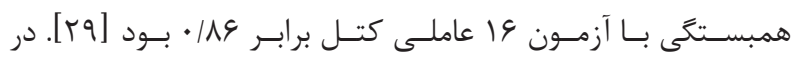

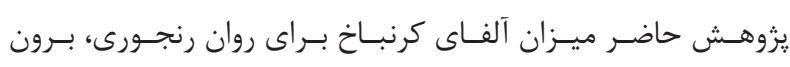

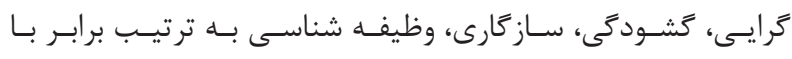

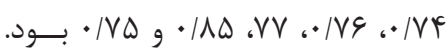

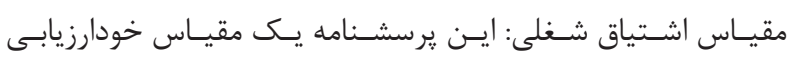

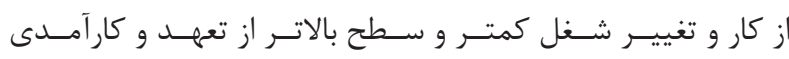

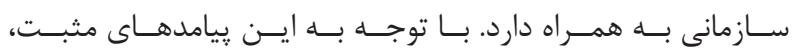

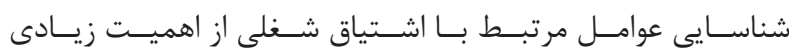

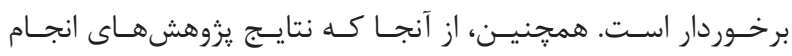

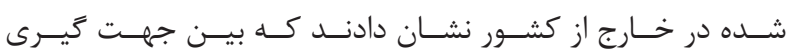

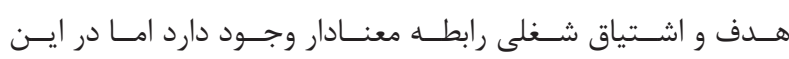

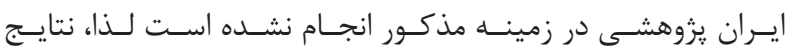

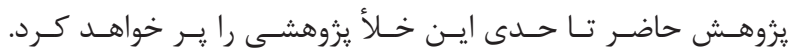

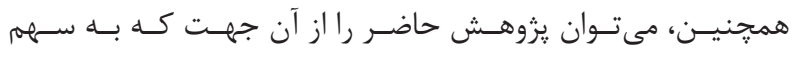

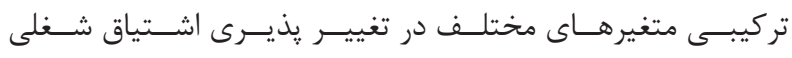

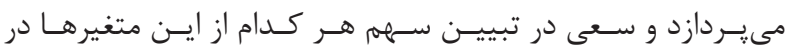

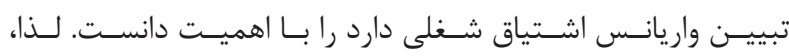

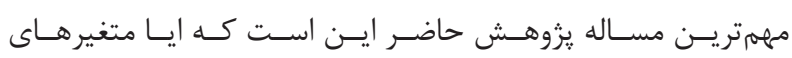

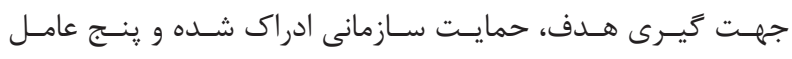

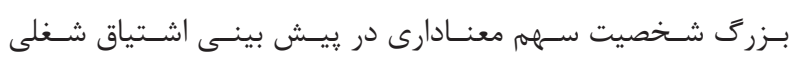
دارنـد؟

\section{روش كار}

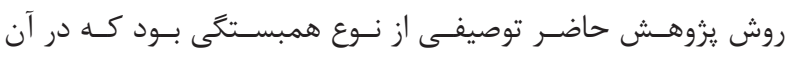

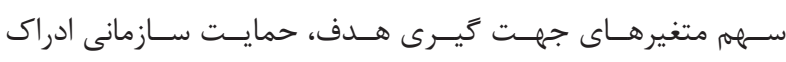

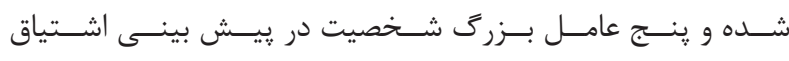

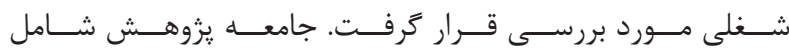

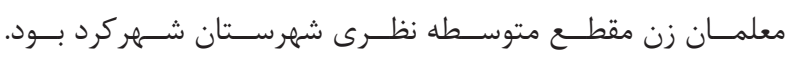

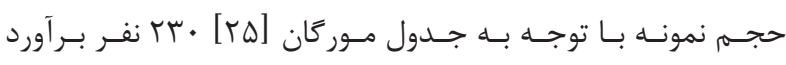

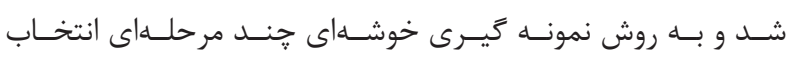

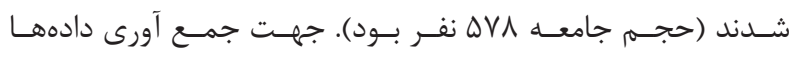

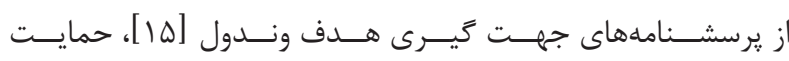

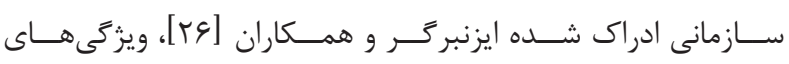

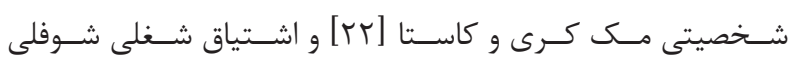

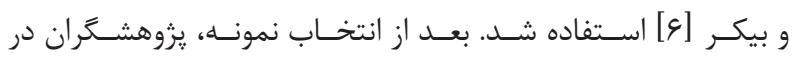

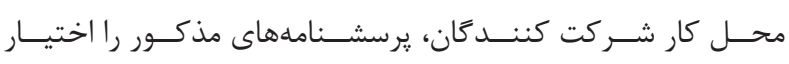

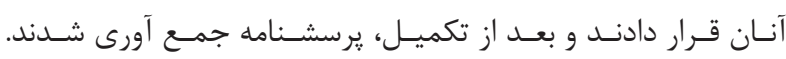

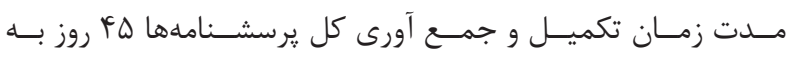

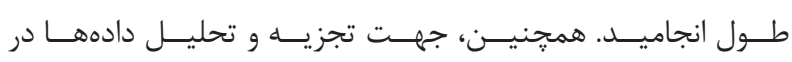

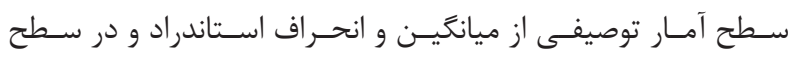

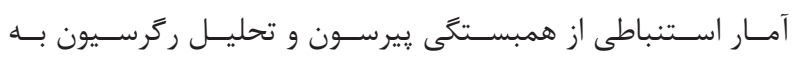

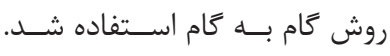

ابزار كرداورى دادهها

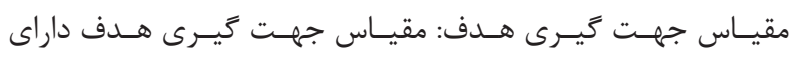

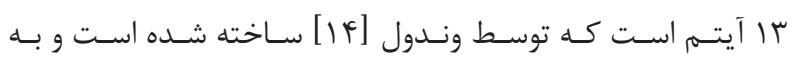

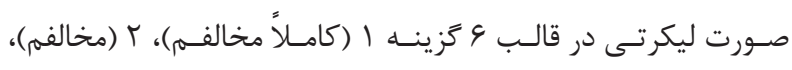




\begin{tabular}{|c|c|c|c|}
\hline \multicolumn{4}{|c|}{ جدول ا: ميانكَين و انحراف استاندراد سن گرووه نمونه } \\
\hline انحراف استاندارد إن & 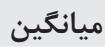 & 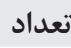 & 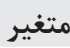 \\
\hline$V / T q$ & rI/Gr & r & 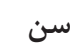 \\
\hline
\end{tabular}

\begin{tabular}{|c|c|c|}
\hline \multicolumn{3}{|c|}{ جدول r: ميانكَين و انحراف استاندارد متغيرهاى اصلى يزوهش } \\
\hline انحراف استاندراد & ميانََين & متغير \\
\hline IV/TV & $\wedge 1 / 1 \wedge$ & اشتياق شغلى \\
\hline \multirow[t]{2}{*}{$\Delta / / q$} & $r r / l l$ & حمايت سازمانى ادراك شده \\
\hline & & جهت عيرى هدف \\
\hline$\Delta / \Delta r$ & $19 / 19$ & يادكيرى \\
\hline r/VG & $1 \Delta / \Delta$ & 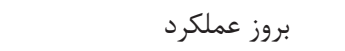 \\
\hline \multirow[t]{2}{*}{ ५/৭६ } & $1 \Delta / 4 \cdot$ & اجتناب عملكرد \\
\hline & & ينج عامل بزرى شخصيت \\
\hline F/QH & rᄉ/l६ & روان رنجورى \\
\hline$r / \cdot 1$ & r & برون كرايى \\
\hline$\Delta / \Delta T$ & 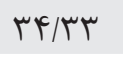 & 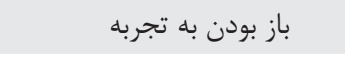 \\
\hline$f / 19$ & rm/Vt & توافق يذيرى \\
\hline$p / r \omega$ & re/my & 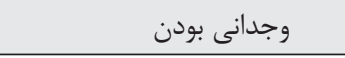 \\
\hline
\end{tabular}

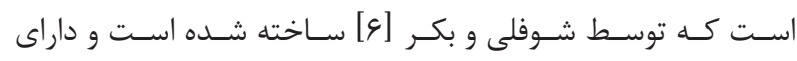

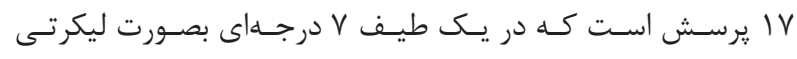

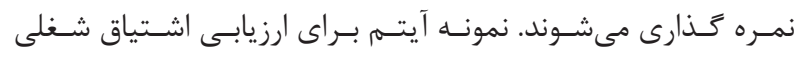

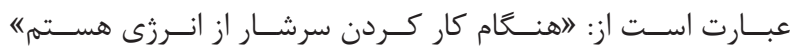

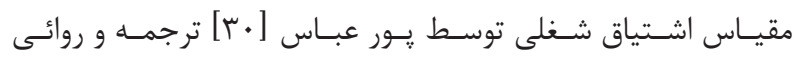

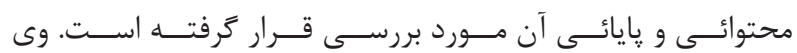

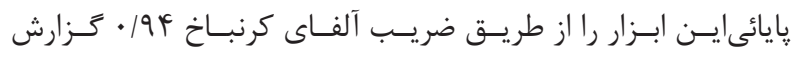

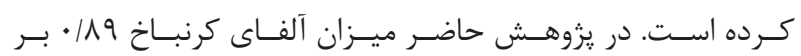
آورد كرديسد.

\section{يافتهها}

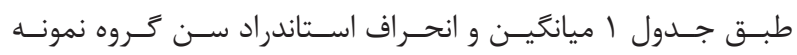

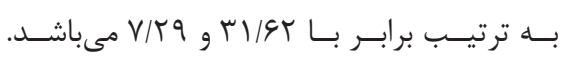

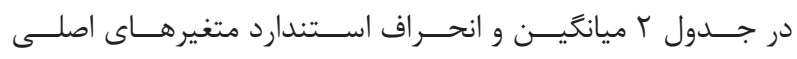

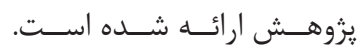

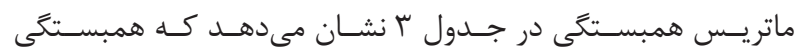

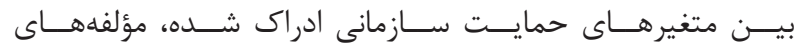

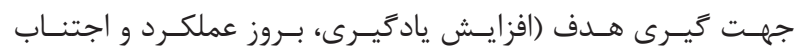

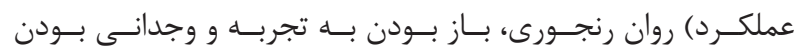

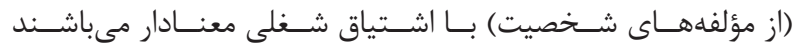

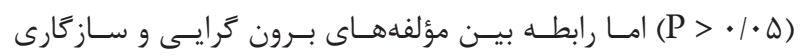

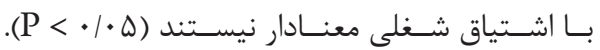

\begin{tabular}{|c|c|c|c|c|c|c|c|c|c|c|}
\hline \multirow[b]{2}{*}{ وبدانى } & \multirow[b]{2}{*}{ باز بودن } & \multirow[b]{2}{*}{ سازَارى } & \multirow[b]{2}{*}{ ترايى برون } & \multirow[b]{2}{*}{ رنجورى روان } & \multirow[b]{2}{*}{ عملكرد } & \multirow[b]{2}{*}{ عملكرود } & \multirow[b]{2}{*}{ يادكيرى } & \multicolumn{3}{|c|}{ جدول "ا: ماتريس همبستخى بين متغيرهاى يزوهش } \\
\hline & & & & & & & & حمايت سازمانى & شغتيلى & متغير \\
\hline & & & & & & & & & 1 & اشتياق شغلى \\
\hline & & & & & & & & 1 & $* \cdot 119$ & شدمايت سازمانى ادراى \\
\hline & & & & & & & 1 & $\cdot / \cdot \Delta$ & $* * / \Delta \Lambda$ & 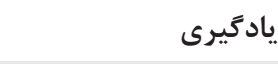 \\
\hline & & & & & & 1 & & $\cdot / \cdot r$ & $* * / \& \Delta$ & 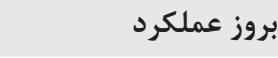 \\
\hline & & & & & 1 & $\cdot / 4 \wedge$ & $\cdot / r \Delta$ & $\cdot / \cdot 1$ & * & 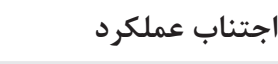 \\
\hline & & & & 1 & $\cdot|r|$ & $\cdot / 4 q$ & $\cdot / 4$ & $\cdot / \cdot \Delta$ & 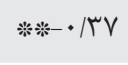 & 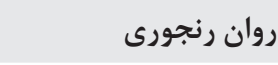 \\
\hline & & & 1 & $\cdot 1 \cdot 9$ & $-\cdot 1 \cdot \cdot 9$ & $\cdot 1 \cdot 9$ & $\cdot / 1 r$ & $\cdot 1 \cdot \wedge$ & $\cdot 111$ & 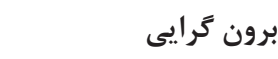 \\
\hline & & 1 & $-\cdot / 11$ & $\cdot / 1 \wedge$ & $\cdot 1 \cdot 1$ & $\cdot / \cdot \uparrow$ &.$/ 1 T$ & $-\cdot \cdot \cdot V$ & $\cdot 111$ & سازگَارى \\
\hline & 1 & $-\cdot / 11$ & $\cdot / 11$ & $\cdot / \cdot \wedge$ & $\cdot / \cdot r$ & $\cdot / 1 \Lambda$ & $-\cdot / \mu \cdot$ & $-\cdot / 14$ & *** & باز بودن به تجربه \\
\hline 1 & $-\cdot / 11$ & $\cdot \mid \Lambda 9$ &.$- / 1 T$ & $\cdot / r \Lambda$ & $\cdot / 11$ & $\cdot / 1 F$ & $\cdot / r \Delta$ & $-\cdot 1 \cdot \Delta$ & $* * / r T$ & وجدانى بودن \\
\hline
\end{tabular}




\begin{tabular}{|c|c|c|c|c|c|c|c|c|}
\hline \multirow{3}{*}{ Adj RY } & \multicolumn{8}{|c|}{ جدول f: تحليل واريانس مدل ركرسيون گَام به گام در تعيين سهمم متغيرهاى پِيش بين در اشتياق شغلى } \\
\hline & & $\mathbf{R}$ & 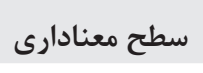 & $\mathbf{F}$ & 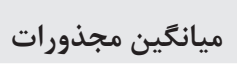 & درجه آزادى & مجموع مجذورات & منابع تغيير \\
\hline & & & $\cdot / \cdot \cdot 1$ & $\| \Delta / \Lambda \Lambda$ & & & & 1 \\
\hline \multirow[t]{4}{*}{ r } & سא/. & $\cdot 1 \Delta \Lambda$ & & & r & 1 & $r r \cdot \Delta / v q$ & رَرسيون \\
\hline & & & & & $191 / 01$ & TTV & $\varphi \Delta \cdot 9 \pi / 1 r$ & باقى مانده \\
\hline & & & & & & rTA & $9 \Lambda \cdot 9 \Lambda / 9 T$ & كل \\
\hline & & & $\cdot 1 \cdot 1$ & $\Lambda \cdot / 9$ & & & & $r$ \\
\hline \multirow[t]{4}{*}{$\cdot|4|$} & $\cdot|4|$ &. $\mid 94$ & & & $|4| 99 / 4 q$ & r & 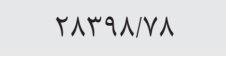 & 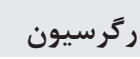 \\
\hline & & & & & IVD/OT & rrg & rqsv./lf & باقى مانده \\
\hline & & & & & & rrA & $9 \Lambda \cdot 9 \Lambda / 9 T$ & كل \\
\hline & & & $\cdot 1 \cdots 1$ & $\Delta 9 / \mu r$ & & & & r \\
\hline \multirow[t]{4}{*}{$\cdot \operatorname{ct}$} &.$/ F F$ & .199 & & & $1 \cdot r \mid / F F$ & r & $r \cdot .9 F / r r$ & رَرسيون \\
\hline & & & & & $18 N / 9$. & TrQ & $r \wedge \cdots r / \Delta \Lambda$ & باقى مانده \\
\hline & & & & & & TrA & $9 \Lambda \cdot 9 \Lambda / 9 r$ & كل \\
\hline & & & $\cdot 1 \cdot \cdot 1$ & $\mathrm{FV} / \cdot \mathrm{q}$ & & & & $p$ \\
\hline \multirow[t]{4}{*}{ - IfF } & $\cdot / 4 \Delta$ & $.19 \mathrm{~V}$ & & & VVVr/G9 & f & $\mathrm{r} .9 F / V$ & رَرسيون \\
\hline & & & & & $19 \Delta / \cdot 9$ & TMF & rGqVY/lD & باقى مانده \\
\hline & & & & & & & $9 \Lambda \cdot 9 \Lambda / 9 T$ & كل \\
\hline & & & $\cdot 1 \cdot \cdot 1$ & ५ //19 & & & & $\Delta$ \\
\hline \multirow[t]{3}{*}{$\cdot / 4 \Delta$} &.$/ 49$ & $\cdot 191$ & & & GTSVIAT & $\Delta$ & ऍ\^ґq/l. & رَرسيون \\
\hline & & & & & $19 T / 49$ & TrT & rGTrq/AT & باقى مانده \\
\hline & & & & & & THA & $9 \Lambda \cdot 9 N / 9 T$ & كل \\
\hline
\end{tabular}

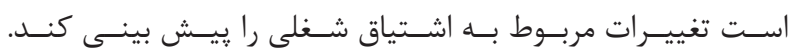

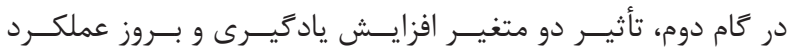

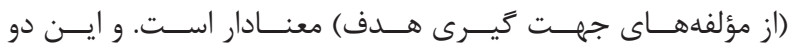

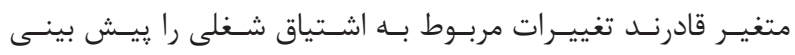

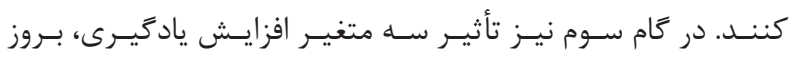

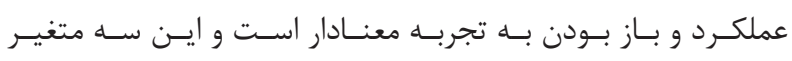

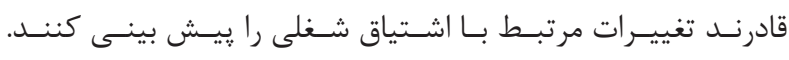

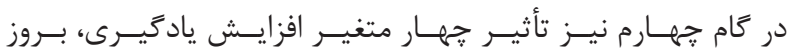

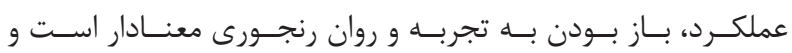

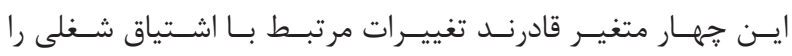

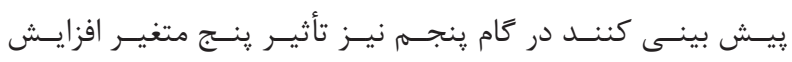

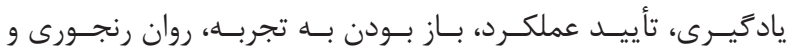

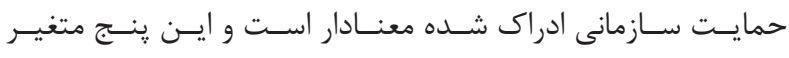

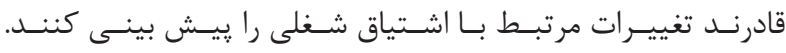

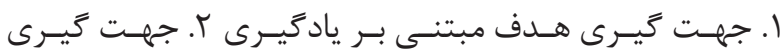

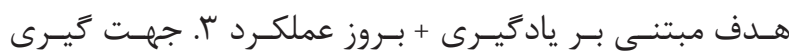

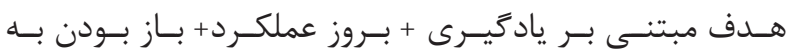

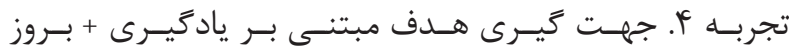

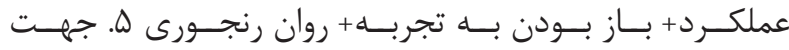

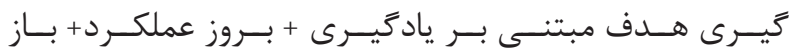

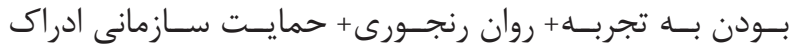

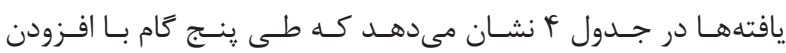

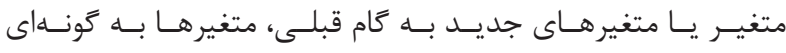

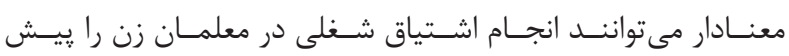

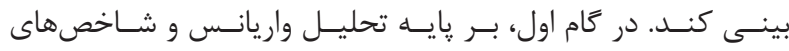

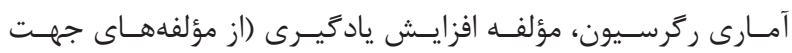

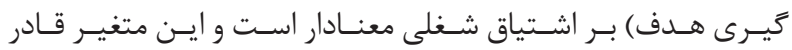




\begin{tabular}{|c|c|c|c|c|}
\hline \multirow[b]{2}{*}{ 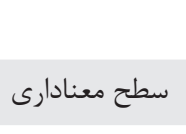 } & \multirow[b]{2}{*}{$\mathrm{t}$} & & \multicolumn{2}{|c|}{ جدول ه: برآورد ضرايب رَرسيون براى متغيرهاى پيش بين اشتياق شغلى } \\
\hline & & Beta & B & متغير هاى بِيش بين \\
\hline & & & & 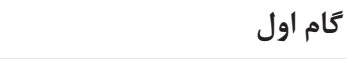 \\
\hline & & & $r \Delta / 1 T$ & مقدار ثابت \\
\hline \multirow[t]{3}{*}{$\cdot 1 \cdot \cdot 1$} & $1 \cdot / V 9$ & $\cdot / \Delta \Lambda$ & $1 / 11$ & افزايش يادَيرى \\
\hline & & & & 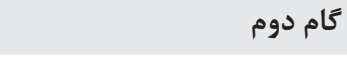 \\
\hline & & & rQ/Ar & مقدار ثابت \\
\hline$\cdot 1 \cdots 1$ & $q / \cdot \vee$ & $\cdot / \& \Lambda$ & $1 / \Delta 1$ & افزايش يادگيرى \\
\hline \multirow[t]{3}{*}{$\cdot / \cdot 1$} & $\Delta / \Delta F$ & $\cdot / \pi 9$ & $1 / \pi 4$ & 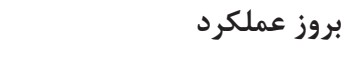 \\
\hline & & & & 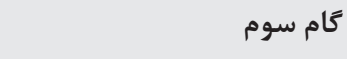 \\
\hline & & & FN/Vq & مقدار ثابت \\
\hline$\cdot 1 \cdot \cdot 1$ & $V / \mu I$ & $\cdot|4|$ & $1 / \pi$ & افزايش يادكيرى \\
\hline$\cdot 1 \cdot \cdot 1$ & $9 / m 4$ & $\cdot / \pi \Delta$ & $1 / 91$ & 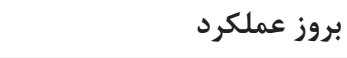 \\
\hline \multirow[t]{3}{*}{$\cdot 1 \cdot r$} & $\mathrm{r} / \mathrm{IF}^{\mathrm{L}}$ & $\cdot / 1 \mathrm{~V}$ & $\cdot / \Delta K$ & 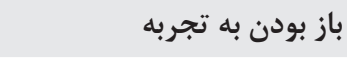 \\
\hline & & & & كام جهارم \\
\hline & & & $r \Delta / \mu^{c}$. & مقدار ثابت \\
\hline$\cdot 1 \cdot \cdot 1$ & G/DT & $\cdot / \pi \wedge$ & $1 / 11$ & افزايش يادگيرى \\
\hline$\cdot 1 \cdot \cdot 1$ & D/FT & $\cdot / \mu$ & I/FT & 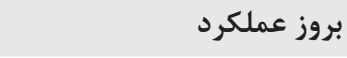 \\
\hline$\cdot 1 \cdot \cdot 1$ & t/AT & $\cdot / 11$ & $\cdot \mid \Delta \Lambda$ & باز بودن به تجربه \\
\hline \multirow[t]{3}{*}{.$/ \cdot 1 \pi$} & $-r / 4 q$ & $-\cdot / 1 r$ & $-\cdot / \Delta T$ & روان رنجورى \\
\hline & & & & كَام ينجم \\
\hline & & & $r r / 91$ & مقدار ثابت \\
\hline$\cdot 1 \cdot \cdot 1$ & $9|9|$ & $\cdot / \mu \Lambda$ & $1 / 19$ & افزايش يادگيرى \\
\hline$\cdot 1 \cdot \cdot 1$ & $\Delta / r \Lambda$ & $\cdot \pi \cdot$ & $1 / 4$. & بروز عملكرد \\
\hline$\cdot 1 \cdot r$ & r/lr & $\cdot / 1 \mathrm{~V}$ & •/Dr & باز بودن به تجربه \\
\hline$\cdot 1 \cdot 1$ & - ( & $-\cdot / 1 r$ & $-\cdot 10$ & روان رنجورى \\
\hline$\cdot / \cdot r$ & $r / l F$ & $\cdot / 1$ & 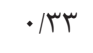 & حمايت سازمانى ادراك شده \\
\hline
\end{tabular}

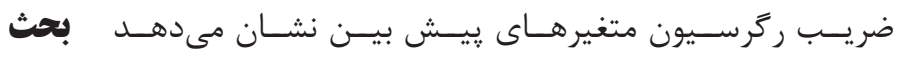

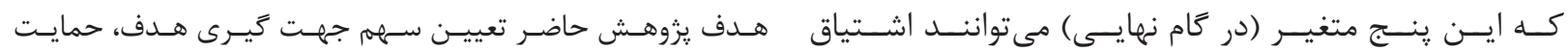

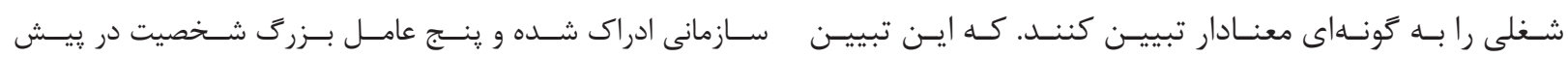

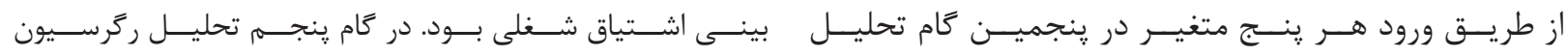

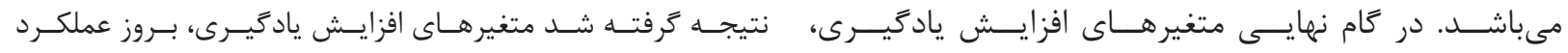

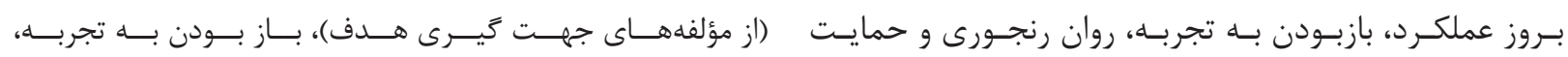

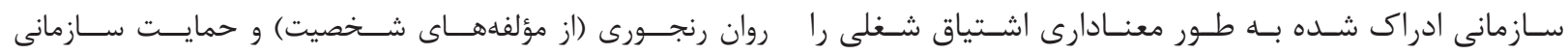

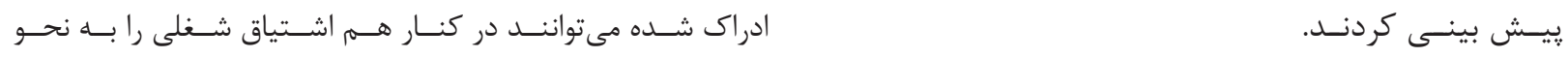




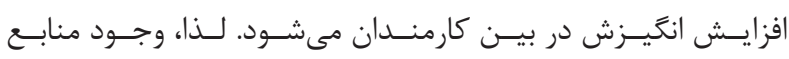

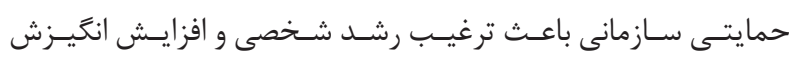

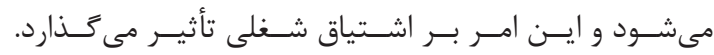

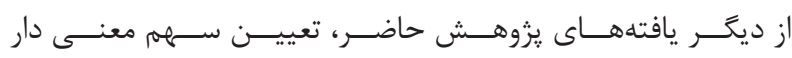

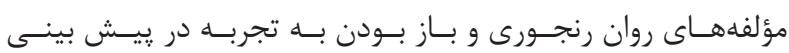

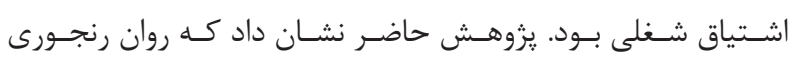

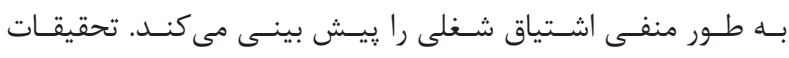

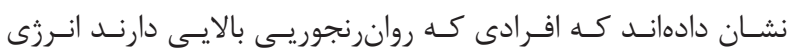

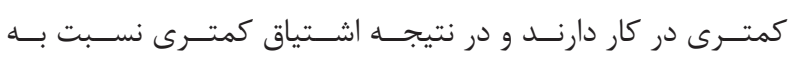

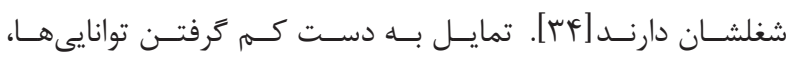

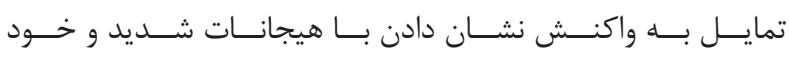

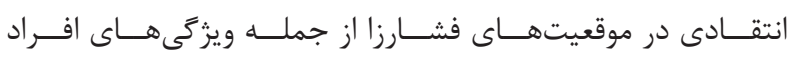

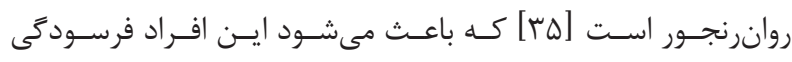

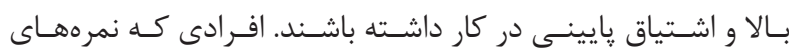

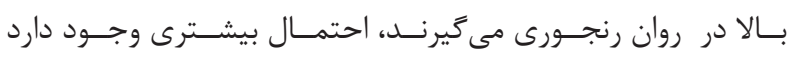

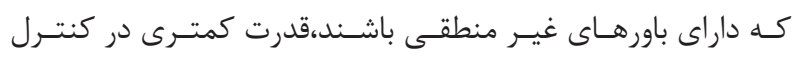

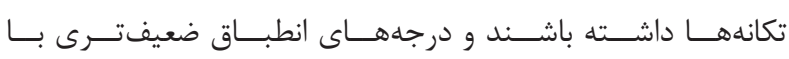

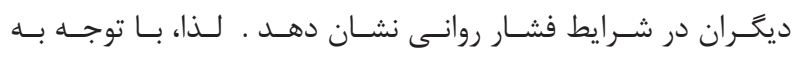

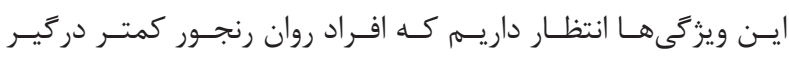

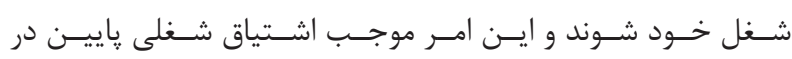

$$
\text { آنـان مى خـــردد. }
$$

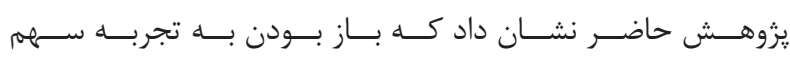

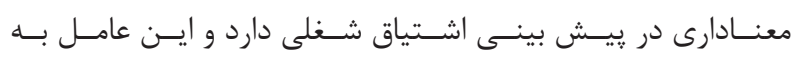

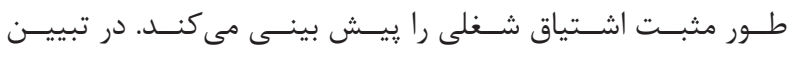

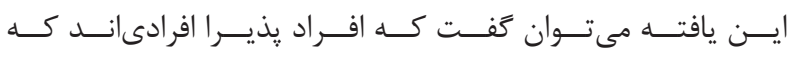

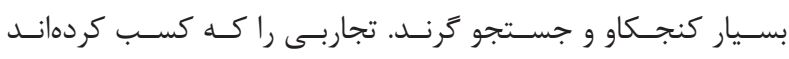

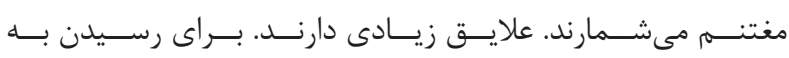

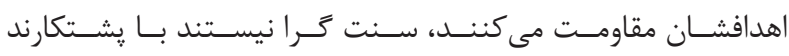

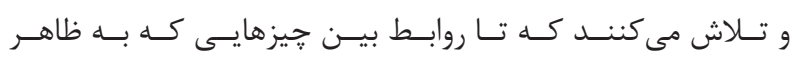

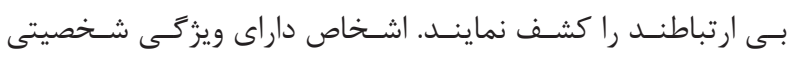

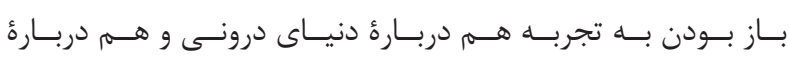

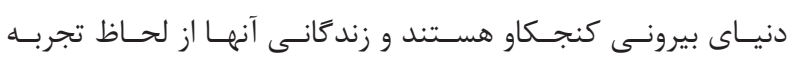

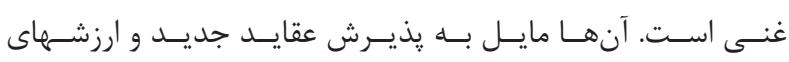

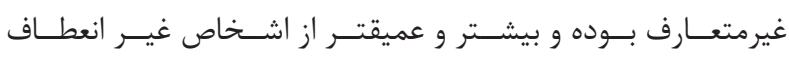

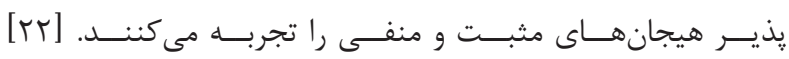

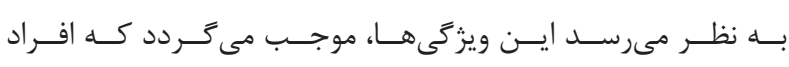

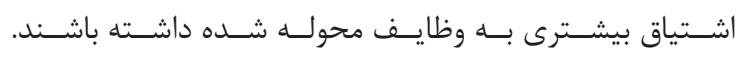

\section{نتيجه كيرى}

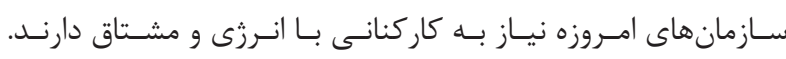

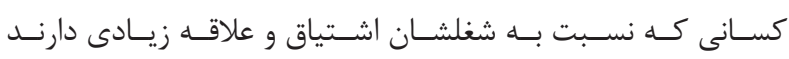

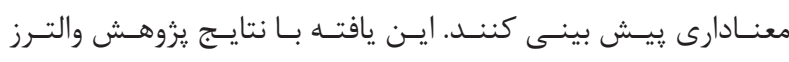

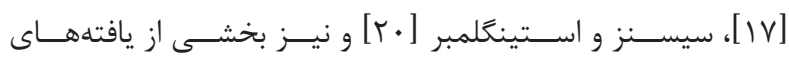

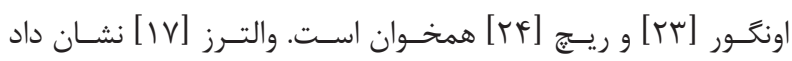

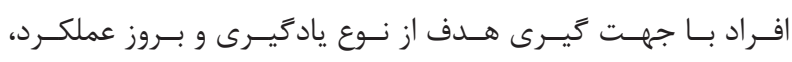

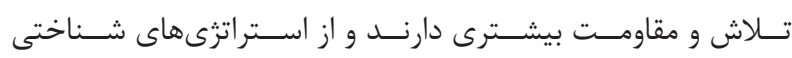

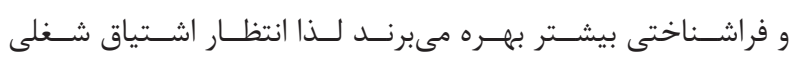

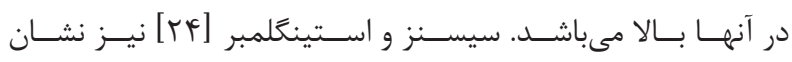

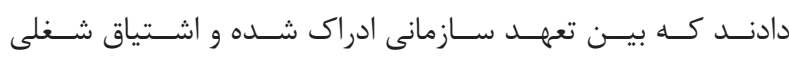

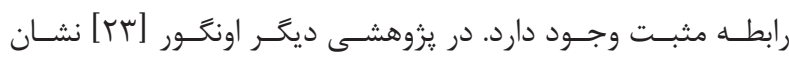

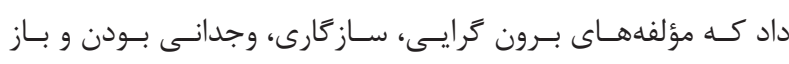

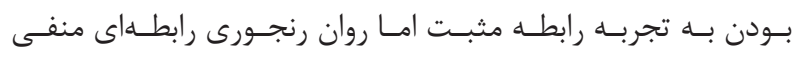

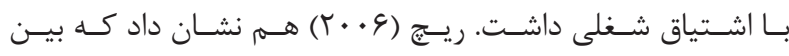

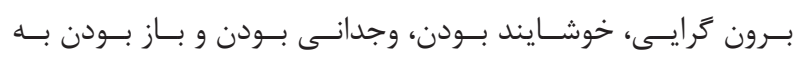

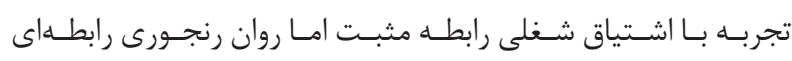
منفـى بـا اشـتياق شــلى دارد.

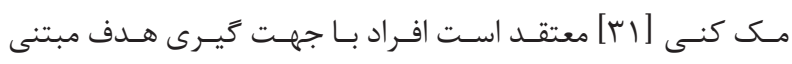

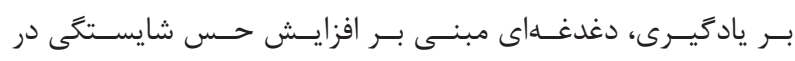

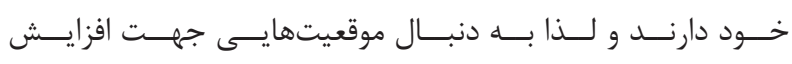

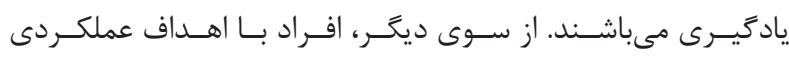

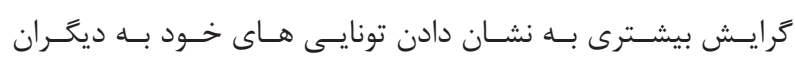

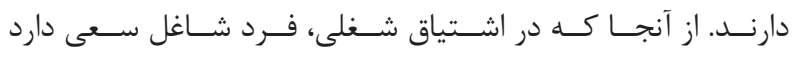

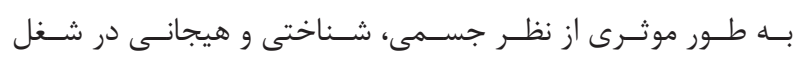

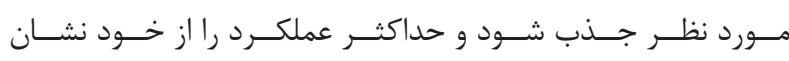

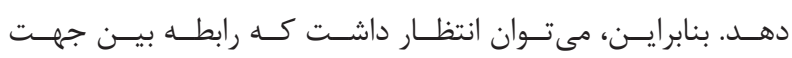

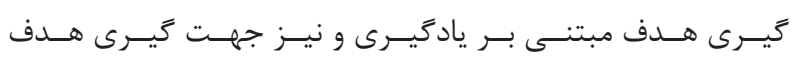

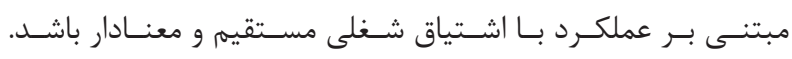

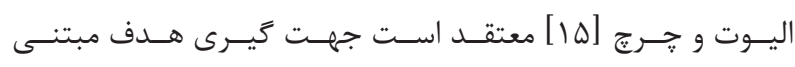

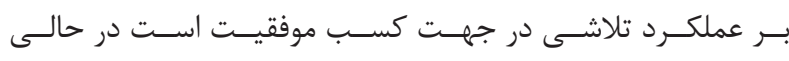

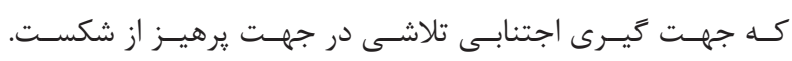

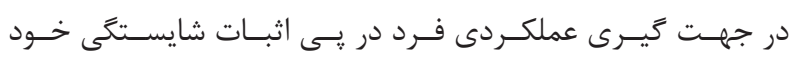

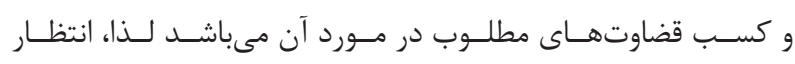

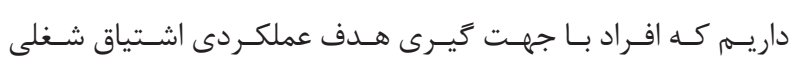
بيشـترى داشـته باشـــند.

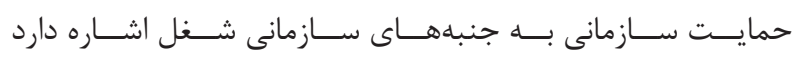

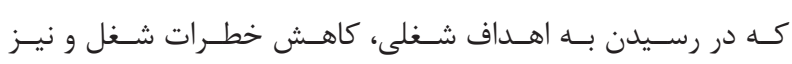

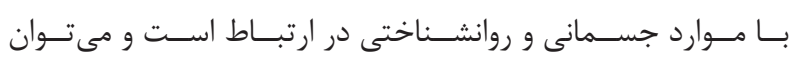

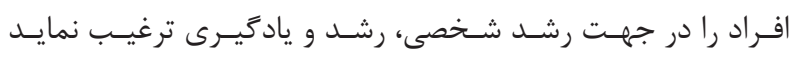

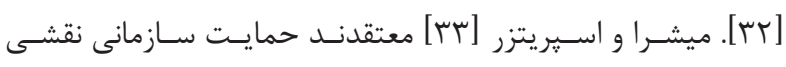

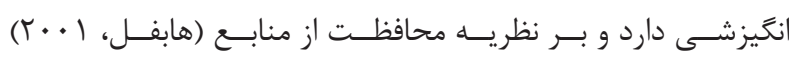

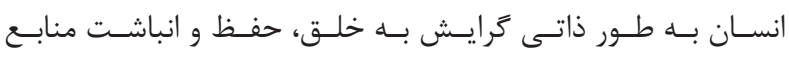

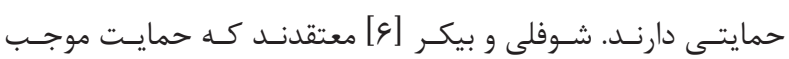




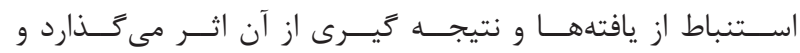

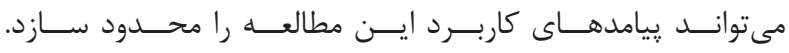

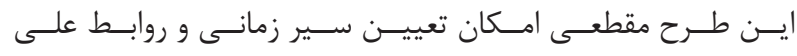

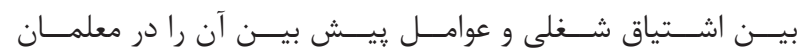

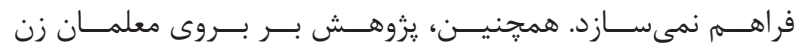

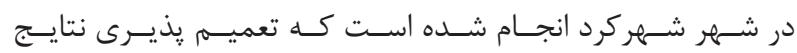

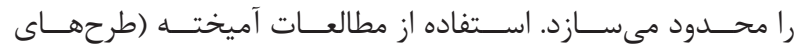

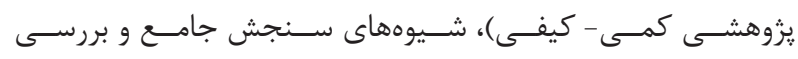

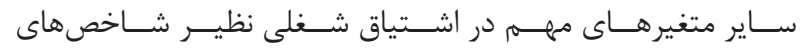

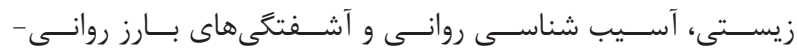

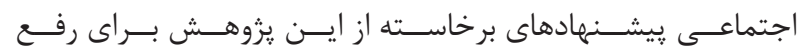

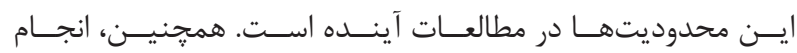

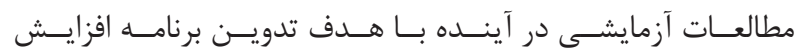

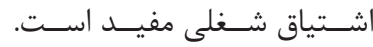

\section{REFERENCES}

1. Schaufeli WB, Salanova M, González-Romá V, Bakker AB. The measurement of engagement and burnout: A two sample confirmatory factor analytic approach. J Happiness Stud. 2002;3(1):71-92.

2. Salanova M, Llorens S, Cifre E, Martínez IM, Schaufeli WB. Perceived collective efficacy, subjective well-being and task performance among electronic work groups an experimental study. Small Group Res. 2003;34(1):43-73.

3. Cooper-Hakim A, Viswesvaran C. The construct of work commitment: testing an integrative framework. Psychol Bull. 2005;131(2):241-59. DOI: 10.1037/0033-2909.131.2.241 PMID: 15740421

4. Nouri A. [Relationship between Work Engagement's Components with Organizational Commitment]. J Mod Indust Organ Psychol. $2011 ; 1(5): 9-15$

5. Csikszentmihalyi M. Flow: The Psychology of Optimal Experience. New York: HarperCollins; 1990.

6. Schaufeli WB, Bakker AB. Job demands, job resources, and their relationship with burnout and engagement: A multi-sample study. J Organ Behav. 2004;25(3):293-315.

7. Molaey M, Mehdad A, Golparvar M. [Relationship of spirituality in workplace, internal motivation and work engagement with task performance]. Knowledge Res Appl Psychol. 2014;15(56):47-55.

8. Miner M, Bickerton G, Dowson M, Sterland S. Spirituality and work engagement among church leaders. Ment Health Religion Cult. 2015;18(1):57-71.

9. Saadi Z, Bavi S. [Relationship Between Work-family conflict family-work with job engagementand marital satisfaction in employees]. J Soc Psychol. 2013;8(27):83.

10. Moen P, Kaduk A, Kossek EE, Hammer L, Buxton OM, O’Donnell E, et al. Is Work-Family Conflict a Multilevel Stressor Linking Job Conditions to Mental Health? Evidence from the Work, Family and Health Network. Res Sociol Work. 2015;26:177-217. DOI: 10.1108/S0277-283320150000026014 PMID: 25866431

11. Hollet-Haudebert S, Mulki JP. Impact of Perfectionism and Self-Efficacy on Job Performance and Work Engagement : Genmes and Boomers. Springer Int. 2015;17(21):175-207. DOI: 10.1007/9783-319-10912-1 6

12. Alagaraja M, Shuck B. Exploring Organizational Alignment-Employee Engagement Linkages and Impact on Individual Performance A Conceptual Model. Hum Resour Dev Rev. 2015;14(1):1737.

13. Kalkan M, Odaci H, Epli Koç H. Self-efficacy, coping with stress and goal-orientation in nurse managers. J Educ Sci. 2011;3:118-25.

14. VandeWalle D. Development and validation of a work domain goal orientation instrument. Educ Psychol Meas. 1997;57(6):995-1015.

15. Elliot AJ, Church MA. A hierarchical model of approach and avoidance achievement motivation. J Pers Soc Psychol. 1997;72(1):21832. DOI: $10.1037 / 0022-3514.72 .1 .218$

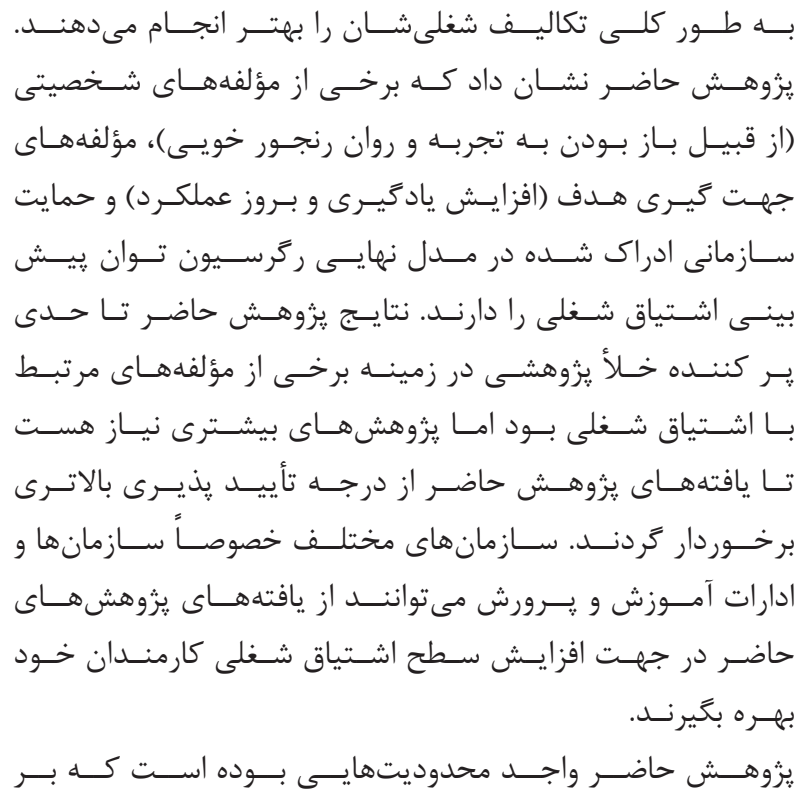

16. Van Knippenberg D, Sleebos E. Organizational identification versus organizational commitment: self-definition, social exchange, and job attitudes. J Organ Behav. 2006;27(5):571-84.

17. Wolters CA. Advancing Achievement Goal Theory: Using Goal Structures and Goal Orientations to Predict Students' Motivation, Cognition, and Achievement. J Educ Psychol. 2004;96(2):236-50. DOI: $10.1037 / 0022-0663.96 .2 .236$

18. Colakoglu U, Culha $\mathrm{O}$, Atay $\mathrm{H}$. The effects of perceived organisational support on employees'affective outcomes: Evidence from the hotel industry. Tourism Hosp Manage. 2010;16(2):125-50.

19. Wayne SJ, Shore LM, Bommer WH, Tetrick LE. The role of fair treatment and rewards in perceptions of organizational support and leader-member exchange. J Appl Psychol. 2002;87(3):590-8. PMID: 12090617

20. Caesens G, Stinglhamber F. The relationship between perceived organizational support and work engagement: The role of self-efficacy and its outcomes. Eur Rev Appl Psychol. 2014;64(5):259-67.

21. Morgan $\mathrm{H}$. Personality traits as risk factors for occupational injury in health care workers. USA: University of Florida.

22. Boyle GJ, Matthews G, Saklofske DH. The Sage Handbook of Personality Theory and Assessment: SAGE Publications; 2008.

23. Ongore O. A Study of Relationship between Personality Traits and Job Engagement. Procedia Soc Behav Sci. 2014;141:1315-9. DOI: $\underline{10.1016 / j . s b s p r o .2014 .05 .226}$

24. Christian MS, Garza AS, Slaughter JE. Work engagement: A quantitative review and test of its relations with task and contextual performance. Pers Psychol. 2011;64(1):89-136.

25. Sadi Pour E. [Research methods in psychology and educational sciences]. Tehran2012.

26. Eisenberger R, Fasolo P, Davis-LaMastro V. Perceived organizational support and employee diligence, commitment, and innovation. J Appl Psychol. 1990;75(1):51-9. DOI: 10.1037/0021-9010.75.1.51

27. Hashemi L, Latifian M. [Relationship between perfectionism and goal orientation in students]. Q J Psychol Stud. 2009;5(3):7.

28. Asgary B, Poursoltani Zarandi H, Aghaei N, Fattahi J. [Relationship of Perceived Organizational Support with Job Performance among Staff of Sport and Youth Head Offices of Western Provinces of Iran]. Shomal J Manage Physiol sport. 2014;1(2):19-28.

29. Jafari Nezaad H, Biyaban Gard E, Hajihasani M. [Investigate the relationship of personality traits and approaches of study with academic achievement in BA students at allameh Tababtaba'i University]. J Educ Psychol. 2013;25(8):8.

30. Pour Abbas A. [Effect of career self efficacy workshop on work engagement of Esfahan university staffs]. Isfahan, Iran: Isfahan University; 2009.

31. Mc Kinney A. Goal orientation: A test of competing models. USA: University of Virginia; 2003. 
32. Demerouti E, Bakker AB, Nachreiner F, Schaufeli WB. The job demands-resources model of burnout. J Appl Psychol. 2001;86(3):499-512. PMID: 11419809

33. Mishra AK, Spreitzer GM. Explaining how survivors respond to downsizing: The roles of trust, empowerment, justice, and work redesign. Acad Manage Rev. 1998;23(3):567-88.

34. Bakker AB, Van der Zee KI, Lewig KA, Dollard MF. The relation- ship between the Big Five personality factors and burnout: a study among volunteer counselors. J Soc Psychol. 2006;146(1):31-50. DOI: 10.3200/SOCP.146.1.31-50 PMID: 16480120

35. Nigg JT, John OP, Blaskey LG, Huang-Pollock CL, Willcutt EG, Hinshaw SP, et al. Big five dimensions and ADHD symptoms: links between personality traits and clinical symptoms. J Pers Soc Psychol. 2002;83(2):451-69. PMID: 12150240 


\title{
The Investigation of the Contribution of Goal Orientation, Percieved Organizational Support and Big Five Personality Traits in Predicting Work Engagement of Female Teachers in Shahrekord, Iran
}

\author{
Mehrdad Hajihasani ${ }^{1{ }^{*}}$, Manijeh Kaveh ${ }^{1}$ \\ ${ }^{1}$ Assistant Professor, Department of Counseling, University of Shahrekord, \\ Shahrekord, Iran \\ * Corresponding author: Mehrdad Hajhasani, Assistant Professor, Department of \\ Counseling, University of Shahrekord, Shahrekord, Iran.E-mail: mehrdadhajihasani@ \\ gmail.com
}

DOI: $10.21859 /$ joe-04031

Received: 11/03/2016

Accepted: 26/08/2016

\section{Keywords:}

\section{Goal Orientation}

Perceived Job Support

Big Five Factor Personality

Traits

Work Engagement

\section{How to Cite this Article:}

Hajhasani M, Kaveh M. The investigation of the contribution of goal orientation, percieved organizational support and Big Five personality traits in predicting work engagement of female teachers in Shahrekord, Iran. J Ergo. 2016;4(2):1-10. DOI: 10.21859 / joe- 04031

(c) 2016 Hamedan University of Medical Sciences.

\section{Abstract}

Introduction: work engagement is a stable and positive state of mind associated with work, which protects against job burnout. This study was conducted to determine the contribution of goal orientation, perceived organizational support and Big Five personality traits in predicting job motivation of female teachers in Shahrekord, Iran.

Methods: The design of the study was correlational-survey. The sample included 230 female teachers in Shahrekord, teaching in high school during 2014-2013. The sample of the study was selected by cluster sampling. To collect data, goal-orientation, perceived organizational support, five factor inventory and job work engagement were administered to the participants.

Results: Correlation and step regression analyses were utilized to analyze the data. Findings of the study showed that there was a significant relationship among goal-orientation, perceived organizational support, Big Five personality traits and work engagement $(\mathrm{P}<$ $0.01)$. Furthermore, multiple regression analysis revealed that linear combination of goal orientation, perceived organizational support and Big Five personality traits could predict work engagement of teachers $(\mathrm{P}<0.01)$.

Conclusions: the present study indicated that different components of personality, goalorientation and perceived organizational support can create work engagement. 